九州大学学術情報リポジトリ

Kyushu University Institutional Repository

\title{
ASYMPTOTIC PROPERTY OF WRAPPED CAUCHY KERNEL DENSITY ESTIMATION ON THE CIRCLE
}

Tsuruta, Yasuhito

Graguate School of Human and Socio-Environment Studies, Kanazawa University

Sagae, Masahiko

School of Economics, Kanazawa University

https://doi. org/10.5109/2232318

出版情報: Bulletin of informatics and cybernetics. 49, pp.1-10, 2017-12. Research Association of Statistical Sciences

バージョン：

権利関係 : 


\section{ASYMPTOTIC PROPERTY OF WRAPPED CAUCHY KERNEL DENSITY ESTIMATION ON THE CIRCLE}

by

Yasuhito Tsuruta and Masahiko SAgAe

Reprinted from the Bulletin of Informatics and Cybernetics Research Association of Statistical Sciences, Vol.49

FUKUOKA, JAPAN

2017 


\title{
ASYMPTOTIC PROPERTY OF WRAPPED CAUCHY KERNEL DENSITY ESTIMATION ON THE CIRCLE
}

\author{
By \\ Yasuhito Tsuruta* and Masahiko SAGAE ${ }^{\dagger}$
}

\begin{abstract}
We discuss theoretical properties of the Wrapped Cauchy (WC) kernel. In this paper, we show that the WC kernel has the convergent rate of $O\left(n^{-2 / 3}\right)$ and holds the asymptotic normality. The rate of the $\mathrm{WC}$ kernel is not better than the rate $O\left(n^{-4 / 5}\right)$ of the von Mises (VM) kernel. However, some numerical experiments show the better behavior of the WC kernel rather than the VM kernel under the condition of the multimodality and/or the heavy tail.
\end{abstract}

Key Words and Phrases: Kernel density estimation, Circular data, Wrapped Cauchy kernel, von Mises kernel, Directional data.

\section{Introduction}

Directional data are represented by an angle $(\theta \in[0,2 \pi), \theta=\theta+2 m \pi, m \in \mathbb{Z})$ or a unit vector $\left(\boldsymbol{x}=(\cos (\theta), \sin (\theta))^{T}\right)$. Some examples of directional data are wind directions and electric power over a period of 24 hours and so on. For the preceding work of the kernel density estimation on the circle; See Hall et al. (1987), Taylor (2008), and Di Marzio et al. (2009, 2011).

Di Marzio et al. (2011) introduced the moments of sin-order $p$ for the kernel on the circle. Its idea is somewhat similar to the moments of order $p$ on the real line. The kernel of sin-order 2 includes the von Mises (VM) kernel, the wrapped Cauchy (WC) kernel and the wrapped normal kernel. Di Marzio et al. (2011) derived asymptotic mean integrated square error (AMISE) via the definition of the kernel functions with sin-order $p$. They show the convergent rate of the VM kernel is $O\left(n^{-4 / 5}\right)$. This rate is equivalent to the convergent rate of the kernels of second order on the real line. However, the convergent rate of the AMISE can not be derived without specifying the kernel.

The VM kernel is called circular normal kernel. However, the VM kernel does not hold the reproductive property (Mardia and Jupp (1999, p44)). This is a big difference from the normal kernel on the line. The WC kernel holds the reproductive property and have more heavy tails than the VM kernel (Mardia and Jupp (1999, p39 and p52)). We explore properties of the WC kernel with respect to those advantages.

We show that the WC kernel has the optimal rate $O\left(n^{-2 / 3}\right)$ of the AMISE and the asymptotic normality. Di Marzio et al. (2011) mentioned the another expression about

\footnotetext{
* Graguate School of Human and Socio-Environment Studies, Kanazawa University Kakumamachi Kanazawa 920-1192 Japan. tsuruta9764@gmail.com

$\dagger$ School of Economics, Kanazawa University Kakumamachi Kanazawa 920-1192 Japan. sagae.masahiko@gmail.com
} 
the asymptotic normality of the circular kernel function. The WC kernel is inferior to the VM kernel with respect to the theoretical aspect of the MISE. However, our numerical experiments show that the WC kernel is superior to the VM kernel under the condition of the multimodal and/or the heavy tail.

\section{Properties of AMISE of a circular kernel density estimation}

Following Di Marzio et al. (2009, 2011), this section briefly refers definitions of the kernel function and properties of the AMISE.

\subsection{Definition}

Definiton 1 (Kernel function)

Let $K_{\kappa}(\theta)$ be a circular kernel function and $\kappa$ be a concentration parameter ( $\kappa$ is a smoothing parameter corresponding to the inverse of bandwidth on the real line), where $K_{\kappa}(\theta):[0,2 \pi) \rightarrow \mathbb{R}$ is such that

(a) It admits an uniformly convergent Fourier series,

$$
K_{\kappa}(\theta)=(2 \pi)^{-1}\left\{1+\sum_{j=1}^{\infty} \gamma_{j}(\kappa) \cos (j \theta)\right\}, \quad \theta \in[0,2 \pi),
$$

where $\gamma_{j}(\theta)$ is strictly monotonic function of $\kappa$,

(b) $\int_{0}^{2 \pi} K_{\kappa}=1$, and if $K_{\kappa}(\theta)$ takes negative values, there exists $0<M<\infty$ such that, $\int_{0}^{2 \pi}\left|K_{\kappa}\right| d \theta \leq M$, for all $\kappa>0$,

(c) $\lim _{\kappa \rightarrow \infty} \int_{\delta}^{2 \pi-\delta}\left|K_{\kappa}(\theta)\right| d \theta=0$, for all $0<\delta<\pi$.

Definiton 2 (Sin-order moment of the circular kernel)

Let $\eta_{j}\left(K_{\kappa}\right):=\int_{0}^{2 \pi} \sin ^{j}(\theta) K_{\kappa}(\theta) d \theta . K_{\kappa}$ of sin-order $p$ is chosen so that,

$$
\eta_{0}\left(K_{\kappa}\right)=1, \quad \eta_{j}\left(K_{\kappa}\right)=0, \quad 0<j<p, \quad \text { and } \quad \eta_{p}\left(K_{\kappa}\right) \neq 0 .
$$

If $\eta_{j}\left(K_{\kappa}\right)$ is $j=2 s(s=1,2, \ldots)$, then $\eta_{j}\left(K_{\kappa}\right)$ is also represented by the sum of Fourier series $\gamma_{j}(\kappa)$ :

$$
\eta_{j}\left(K_{\kappa}\right)=\frac{1}{2^{2 s-1}}\left[\left(\begin{array}{c}
j-1 \\
j / 2
\end{array}\right)+\sum_{s=1}^{j / 2}(-1)^{j+s}\left(\begin{array}{c}
j \\
j / 2+s
\end{array}\right) \gamma_{2 s}(\kappa)\right]
$$

Definiton 3 (Kernel density estimator)

Let $\Theta_{1}, \ldots, \Theta_{n}$ be random sample from the unknown circular density $f(\theta)$. Given a circular kernel $K_{\kappa}$, the kernel estimator of $f$ is defined as,

$$
\hat{f}(\theta ; \kappa):=\frac{1}{n} \sum_{i=1}^{n} K_{\kappa}\left(\theta-\Theta_{i}\right)
$$




\subsection{Theoretical properties}

Theorem 1 (AMISE)

Under the following conditions:

(A) $\kappa$ increases as $n \rightarrow \infty$. for each $j \in \mathbb{Z}^{+}, \lim _{n \rightarrow \infty} \gamma_{j}(\kappa)=1$,

(B) $\lim _{n \rightarrow \infty} n^{-1} \sum_{j=1}^{\infty} \gamma_{j}^{2}(\kappa)=0$,

(C) $f^{\prime \prime}$ is continuous and square-integrable,

the second sin-order kernel has,

$$
A M I S E[\hat{f}(\cdot ; \kappa)]=\frac{\eta_{2}^{2}\left(K_{\kappa}\right)}{4} R\left(f^{\prime \prime}\right)+\frac{R\left(K_{\kappa}\right)}{n},
$$

where $\int_{0}^{2 \pi}\{g(\theta)\}^{2} d \theta=R(g), \eta_{2}\left(K_{\kappa}\right)=\frac{1}{2}\left(1-\gamma_{2}(\kappa)\right)$ and $R\left(K_{\kappa}\right)=\left(1+2 \sum_{i=1}^{\infty} \gamma_{j}^{2}(\kappa)\right) /(2 \pi)$. The right-hand side terms of (2) correspond to $I S B$ and $I V$, respectively.

The convergent rate of AMISE can not be derived without choosing specific kernel, since (2) depends on the kernel function $K_{\kappa}$. Di Marzio et al. (2011) derived AMISE of the VM kernel.

The VM kernel is defined as,

$$
K_{\kappa}(\theta):=\frac{1}{2 \pi I_{0}(\kappa)} \exp \{\kappa \cos \theta\}, 0<\kappa<\infty,
$$

where $I_{p}(\kappa)$ denotes the modified Bessel function of the first kind with order $p$. The characteristic function of VM kernel is defined as,

$$
\phi_{p}=\frac{I_{p}(\kappa)}{I_{0}(\kappa)} \quad p=0, \pm 1, \ldots, \pm n, \cdots
$$

Proposition 1 (AMISE of VM kernel)

Noting $\gamma_{j}(\kappa)=I_{j}(\kappa) / I_{0}(\kappa)$, the AMISE of the VM kernel is given as the following form :

$$
A M I S E_{V M}[\hat{f}(\cdot ; \kappa)]=\frac{1}{16}\left\{1-\frac{I_{2}(\kappa)}{I_{0}(\kappa)}\right\}^{2} R\left(f^{\prime \prime}\right)+\frac{1+2 \sum_{j=1}^{\infty}\left\{I_{j}(\kappa) / I_{0}(\kappa)\right\}^{2}}{2 n \pi}
$$

Under the condition that $\kappa$ is sufficiently large, the asymptotic form (3) reduces,

$$
A M I S E_{V M}[\hat{f}(\cdot ; \kappa)]=\frac{1}{4 \kappa^{2}} R\left(f^{\prime \prime}\right)+\frac{\kappa^{1 / 2}}{2 n \pi^{1 / 2}} .
$$

We obtain the optimum $\kappa^{*}$ :

$$
\kappa^{*}=\left(2 \pi^{1 / 2} R\left(f^{\prime \prime}\right) n\right)^{2 / 5}
$$

From (4) and (5), AMISE $\mathrm{VM}_{\mathrm{VM}}=O\left(n^{-4 / 5}\right)$ is derived. Note that that $O\left(n^{-4 / 5}\right)$ is equivalent to the convergent rate of the second-order kernels on the real line. 


\section{Properties of the Wrapped Cauchy kernel}

The WC kernel is defined as,

$$
K_{\rho}(\theta)=\frac{1}{2 \pi} \frac{1-\rho^{2}}{1+\rho^{2}-2 \rho \cos (\theta)}, 0<\rho \leq 1,
$$

where $\rho$ is concentration parameter. The characteristic function of the WC kernel is equal to,

$$
\phi_{p}=\rho^{|p|}, \quad p=0, \pm 1, \ldots, \pm n, \cdots .
$$

Theorem 2 (AMISE of WC kernel)

The AMISE of the WC kernel is given as the follows,

$$
A M I S E_{W C}[\hat{f}(\cdot ; \rho)]=\frac{\left\{1-\rho^{2}\right\}^{2} R\left(f^{\prime \prime}\right)}{16}+\frac{1}{n \pi\left(1-\rho^{2}\right)} .
$$

Let be $1-\rho^{2}=h, 0 \leq h<1$. Then, (6) is expressed as,

$$
\operatorname{AMISE}_{W C}[\hat{f}(\cdot ; h)]=\frac{h^{2} R\left(f^{\prime \prime}\right)}{16}+\frac{1}{n \pi h} .
$$

See Appendix-A for the details.

In the similar way of Propositon 1 . we obtain the optimum $h^{*}$ :

$$
h^{*}=\left(\frac{8}{\pi R\left(f^{\prime \prime}\right) n}\right)^{1 / 3},
$$

under the condition of $n>8\left(\pi R\left(f^{\prime \prime}\right)\right)^{-1}$. The optimal following convergent rate of AMISE $_{W C}$ is given by (7) and (8) as $n$ is sufficiently large:

$$
\operatorname{AMISE}_{\mathrm{WC}}=O\left(n^{-2 / 3}\right) .
$$

We should use $\rho^{*}$ which minimize (6) as the practically optimal concentrating parameter, since (8) is larger than one if $n \leq 8\left(\pi R\left(f^{\prime \prime}\right)\right)^{-1} \cdot \rho^{*}$ is given (7) and (8) as follows,

$$
\rho^{*}=\arg \min _{0<\rho \leq 1}\left\{\operatorname{AMISE}_{\mathrm{WC}}[\hat{f}(\cdot ; \rho)]\right\} .
$$

We put $\hat{f}_{\rho}$ as $\hat{f}_{h}$ with $h=\left(1-\rho^{2}\right)$.

Theorem 3 (Asymptotic Normality)

$$
\text { Let be } \Theta_{1}, \Theta_{2}, \cdots \Theta_{n} \stackrel{i . i . d .}{\sim} f(\theta), h=c n^{-\alpha} \text { and } 0 \leq h<1 \text { If } \alpha>1 / 3 \text {, then }
$$

$$
\sqrt{n h}\left[\hat{f}_{h}(\theta)-f(\theta)\right] \stackrel{d}{\longrightarrow} N(0, f(\theta) / \pi), \quad n \rightarrow \infty .
$$

See Appendix-B for the details.

The optimal convergent rate $O\left(n^{-2 / 3}\right)$ of the AMISE of the WC kernel differs from the rate $O\left(n^{-4 / 5}\right)$ of the VM kernel, although Both the WC kernel and the VM kernel are the second sin-order kernel. The Cauchy kernel on the real line has the 
optimal convergent rate $O\left(n^{-2 / 3}\right)$ by Davis (1975). The rate $O\left(n^{-2 / 3}\right)$ of the WC kernel correspond to the rates of kernel family of order 0 such as the histogram and the Cauchy kernel.

We consider that the corespondence between the characteristic functions of the WC kernel and the Cauchy kernel causes the correspondence between the two rates. Mardia and Jupp (1999, p48 (3.5.59)) said the characteristic function of the WC Kernel corresponds that of Cauchy kernel $\phi(p)=e^{-a|p|}$. However, each of the inversion theorem is different. The inversion theorem of the WC kernel is Fourier series expansion, while that Cauchy kernel is Fourier transform.

\section{Simulation}

The optimal concentration parameter depends on $R\left(f^{\prime \prime}\right)$. The plug-in rule is the procedure to estimate the optimal concentration parameter with $\hat{R}\left(f^{\prime \prime}\right)$ as an estimator for $R\left(f^{\prime \prime}\right)$. The simplest rule among some plug-in rules is the procedure to assume that the true density $f$ is the von Mises density $f_{\mathrm{VM}}$. The simplest plug-in rule uses $\hat{R}\left(f_{\mathrm{VM}}^{\prime \prime}(\cdot ; \hat{\kappa})\right)$ as the estimator for $R\left(f^{\prime \prime}\right)$, where $\kappa$ is the concentration parameter of $f_{\mathrm{VM}}$ and $\hat{\kappa}$ is the maximum likelihood estimator of $\kappa . \hat{R}\left(f_{\mathrm{VM}}^{\prime \prime}(\cdot ; \hat{\kappa})\right)$ corresponds to the following form:

$$
\hat{R}\left(f_{\mathrm{VM}}^{\prime \prime}(\cdot ; \hat{\kappa})\right)=\frac{\hat{\kappa}\left[3 \hat{\kappa} I_{2}(2 \hat{\kappa})+2 I_{1}(2 \hat{\kappa})\right]}{8 \pi I_{0}^{2}(\hat{\kappa})} .
$$

The normal procedure of plug-in rule is shown as,

i) Estimate $\hat{\kappa}$ from the sample and calculate $\hat{R}\left(f_{\mathrm{VM}}^{\prime \prime}(\cdot ; \hat{\kappa})\right)$ from $\hat{\kappa}$,

ii) WC kernel: Estimate $\rho^{*}$ which minimize $(6)$ by substituting $\hat{R}\left(f_{\mathrm{VM}}^{\prime \prime}(\cdot ; \hat{\kappa})\right)$ for $R\left(f^{\prime \prime}\right)$ in $(6)$,

iii) VM kernel : Estimate $\kappa^{*}$ by substituting $\hat{R}\left(f_{\mathrm{VM}}^{\prime \prime}(\cdot ; \hat{\kappa})\right)$ for $R\left(f^{\prime \prime}\right)$ in $(5)$.

The two simulations are given as,

Experiment-A (mixture of the von Mises density)

1. The WC kernel:

(a) Let a true density be a mixture of the von Mises density:

$$
f(\theta)_{\mathrm{MVM}}=\frac{1}{2} f_{\mathrm{VM} 1}\left(\theta ; \mu_{1}, \kappa_{1}\right)+\frac{1}{2} f_{\mathrm{VM} 2}\left(\theta ; \mu_{2}, \kappa_{2}\right),
$$

where let be $\mu_{1}=\pi / 2, \mu_{2}=3 \pi / 2$ and $\kappa_{1}=\kappa_{2}=\kappa$. Generate the random sample of the size $n$ distributed as (13),

(b) Estimate $\rho^{*}$ by the plug-in rule,

(c) Let ISE $=\int_{0}^{2 \pi}\{\hat{f}(\theta ; \rho)-f(\theta)\}^{2} d \theta$, Let $\overline{\operatorname{ISE}}(\hat{f}(\cdot ; \rho))$ be the numerical integration of ISE. Calculate $\overline{\operatorname{ISE}}(\hat{f}(\cdot ; \rho))$,

(d) Repeat (a)-(c) 1000 times and compute $\overline{\operatorname{MISE}}(\hat{f}(\cdot ; \rho))=\sum_{i=1}^{1000} \overline{\operatorname{ISE}}_{i}(\hat{f}(\cdot ; \rho)) / 1000$.

2. The VM kernel :

With the same procedures as (a) - (d) of the WC kernel, compute $\overline{\operatorname{MISE}}\left(\hat{f}\left(\cdot ; \kappa^{*}\right)\right)$, 
3. Calculate $\overline{\operatorname{MISE}}\left(\hat{f}\left(\cdot ; \kappa^{*}\right)\right)-\overline{\operatorname{MISE}}\left(\hat{f}\left(\cdot ; \rho^{*}\right)\right)$, where it round off decimal point forth place.

\section{Experiment-B (wrapped Cauchy density)}

1. Let a true density be a wrapped Cauchy density given by,

$$
f_{w c}(\theta)=\frac{1}{2 \pi} \frac{1-\rho^{2}}{1+\rho^{2}-2 \rho \cos (\theta-\mu)},
$$

where directional mean $\mu=\pi$.

2. Calculate $\overline{\operatorname{MISE}}\left(\hat{f}\left(\cdot ; \kappa^{*}\right)\right)-\overline{\operatorname{MISE}}\left(\hat{f}\left(\cdot ; \rho^{*}\right)\right)$ in the same procedures as ExperimentA.

Table 1: $\overline{\operatorname{MISE}}\left(\hat{f}_{\kappa^{*}}\right)-\overline{\operatorname{MISE}}\left(\hat{f}_{\rho^{*}}\right) . n$ is sample size, $\kappa$ is represented as concentrate parameter of true density $f$, which is a mixture of von Mises density.

\begin{tabular}{|c|cccccc|}
\hline & $n=10$ & 50 & 100 & 200 & 500 & 1000 \\
\hline$\kappa=0.3$ & 0.011 & 0.002 & 0.001 & 0 & 0 & 0 \\
0.5 & 0.012 & 0.002 & 0.001 & 0 & 0 & 0 \\
0.7 & 0.011 & 0.002 & 0.001 & 0 & 0 & 0 \\
1 & 0.012 & 0.001 & 0 & 0 & 0 & 0 \\
2 & 0.007 & -0.004 & -0.005 & -0.005 & -0.005 & -0.005 \\
5 & -0.017 & -0.023 & -0.025 & -0.024 & -0.024 & -0.023 \\
10 & -0.032 & -0.036 & -0.037 & -0.037 & -0.037 & -0.037 \\
15 & -0.037 & -0.044 & -0.043 & -0.042 & -0.042 & -0.042 \\
20 & -0.039 & -0.045 & -0.045 & -0.045 & -0.048 & -0.046 \\
\hline
\end{tabular}

Table 2: $\overline{\operatorname{MISE}}\left(\hat{f}_{\kappa^{*}}\right)-\overline{\operatorname{MISE}}\left(\hat{f}_{\rho^{*}}\right) . n$ is sample size, $\rho$ is represented as concentrate parameter of true density $f$, which is a wrapped Cauchy density.

\begin{tabular}{|c|c|c|c|c|c|}
\hline & $n=50$ & 100 & 200 & 500 & 1000 \\
\hline$\rho=0.5$ & -0.015 & -0.009 & -0.006 & -0.003 & -0.002 \\
\hline 0.7 & -0.011 & -0.006 & -0.003 & -0.001 & -0.001 \\
\hline 0.8 & 0.007 & 0.012 & 0.013 & 0.011 & 0.009 \\
\hline 0.9 & 0.133 & 0.141 & 0.134 & 0.112 & 0.093 \\
\hline
\end{tabular}

The result of Table 1 indicates that the WC kernel is superior to the VM kernel under that $n$ is not sufficiently large $(n \leq 100)$ and $\mathrm{f}$ has the mixture of the von Mises (the multimodal) and $\kappa \leq 2$. This difference between the WC kernel and the VM kernel tends to become smaller as $n$ become larger.

The result of Table 2 shows that WC kernel has better than the VM kernel if $n \leq 1000$ and $\mathrm{f}$ has the wrapped Cauchy (the heavy tail) and $\rho \geq 0.8$. Particularly, the WC kernel has very better behavior about the top of $f$ than the VM kernel even if $n$ is large. 


\section{Conclusion}

This paper shows that the WC kernel has the convergent rate $O\left(n^{-2 / 3}\right)$ of the AMISE and the asymptotic normality. However, the convergent rate of the VM kernel is $O\left(n^{-4 / 5}\right)$. In other words, the rate of the WC kernel and that of the VM kernel do not equal in spite of the same second sin-order kernel.

The convergent rate of the AMISE of the WC kernel corresponds to the rate of Cauchy kernel, since the characteristic function of the WC kernel corresponds to the one of Cauchy kernel.

The WC kernel is not better than the VM kernel with respect to the AMISE convergent rate. However, the results of our simulations show that the WC kernel is superior to the VM kernel if a true density $f$ has the multimodal and/or the heavy tail.

\section{Acknowledgements}

I would like to thank the editor and the referee for the helpful comments. This work was supported by JSPS KAKENHI Grant Number 16K00043. 


\section{Appendix}

\subsection{Appendix-A}

Proof of Theorem 2. The WC kernel has $\gamma_{j}(\rho)=\rho^{j}$ and $\eta_{2}\left(K_{\rho}\right)=\left(1-\rho^{2}\right) / 2$. Since for small values of $u, \sin (u) \simeq u$, we use the expansion $f(\theta+u) \simeq f(\theta)+$ $f^{\prime}(\theta) \sin (u)+f^{\prime \prime}(\theta) \sin ^{2}(u) / 2+O\left(\sin ^{3}(u)\right)$.

$$
\begin{aligned}
\mathrm{E}_{f}\left[K_{\rho}(\theta-Y)\right] & =\int K_{\rho}(\theta-y) f(y) d y \\
& =\int K_{\rho}(u) f(\theta+u) d u \\
& =\int K_{\rho}(u)\left[f(\theta)+f^{\prime}(\theta) \sin (u)+f^{\prime \prime}(\theta) \sin ^{2}(u) / 2+O\left(\sin ^{3}(u)\right)\right] \\
& =f(\theta)+\frac{1}{2} \eta_{2}\left(K_{\rho}\right) f^{\prime \prime}(\theta)+o(1) \\
& =f(\theta)+\frac{1}{4}\left(1-\rho^{2}\right) f^{\prime \prime}(\theta)+o(1) \\
R\left(K_{\rho}\right) & =\frac{1+2 \sum_{j=1}^{\infty}\left(\rho^{j}\right)^{2}}{2 \pi} \\
& =\frac{1+\frac{2 \rho^{2}}{1-\rho^{2}}}{2 \pi} \\
& =\frac{1+\rho^{2}}{2 \pi\left(1-\rho^{2}\right)} \\
& =\frac{1}{\pi\left(1-\rho^{2}\right)}-\frac{1}{2 \pi} .
\end{aligned}
$$

It follows from (15) that

$$
\begin{aligned}
n^{-1} \operatorname{Var}_{f}\left[K_{\rho}(\theta-Y)\right] & =n^{-1}\left\{E_{f}\left[K_{\rho}^{2}(\theta-Y)\right]-\mathrm{E}_{f}\left[K_{\rho}(\theta-Y)\right]^{2}\right\} \\
& =n^{-1}\left[\frac{1}{\pi\left(1-\rho^{2}\right)}-\frac{1}{2 \pi}\right]\{f(\theta)+o(1)\}-n^{-1}[f(\theta)+o(1)] \\
& =\frac{f(\theta)}{\pi n\left(1-\rho^{2}\right)}+o\left(n^{-1}\left(1-\rho^{2}\right)^{-1}\right) .
\end{aligned}
$$

\subsection{Appendix-B}

Proof of Theorem 3. Put $h=\left(1-\rho^{2}\right)$, Write (14) and (16) as,

$$
\begin{gathered}
\mathrm{E}_{f}\left[K_{h}\left(\theta-\Theta_{1}\right)\right] \simeq f(\theta)+\frac{1}{4} h f^{\prime \prime}(\theta)+o(1), \\
\operatorname{Var}_{f}\left[K_{h}\left(\theta-\Theta_{1}\right)\right]=\frac{f(\theta)}{\pi h}+o\left(h^{-1}\right) . \\
\sqrt{n h}\left[\hat{f}_{h}(\theta)-f(\theta)\right]=\sqrt{n h}\left\{\hat{f}_{h}(\theta)-\mathrm{E}\left[\hat{f}_{h}(\theta)\right]\right\}+\sqrt{n h} \operatorname{bias}\left[\hat{f}_{h}(\theta)\right] .
\end{gathered}
$$


The first term of (19) is equal to,

$$
\begin{gathered}
\sqrt{n h}\left\{\hat{f}_{h}(\theta)-\mathrm{E}_{f}\left[\hat{f}_{h}(\theta)\right]\right\}=\sqrt{n}\left\{n^{-1} \sum_{i=1}^{n} h^{1 / 2} K_{h}\left(\theta-\Theta_{i}\right)-\mathrm{E}_{f}\left[h^{1 / 2} K_{h}\left(\theta-\Theta_{1}\right)\right]\right\} \\
\mathrm{E}_{f}\left[h^{1 / 2} K_{h}\left(\theta-\Theta_{1}\right)\right] \\
=h^{1 / 2} \mathrm{E}_{f}\left[K_{h}\left(\theta-\Theta_{1}\right)\right] \\
\simeq h^{1 / 2}\left[f(\theta)+\frac{f^{(\prime \prime)}(\theta)}{4} h\right]
\end{gathered}
$$

It is shown that $0 \leq\left|\mathrm{E}\left[h^{1 / 2} K_{h}\left(\theta-\Theta_{1}\right)\right]\right|<\infty$ from $(21)$.

From (18), We obtain the following form:

$$
\begin{aligned}
\operatorname{Var}_{f}\left[h^{1 / 2} K_{h}\left(\theta-\Theta_{1}\right)\right] & =h \operatorname{Var}\left[K_{h}\left(\theta-\Theta_{1}\right)\right] \\
& =h\left[\frac{f(\theta)}{\pi h}+o\left(h^{-1}\right)\right] \\
& =\frac{f(\theta)}{\pi}+o(1) \\
& \simeq \frac{f(\theta)}{\pi} .
\end{aligned}
$$

It is shown that $\operatorname{Var}_{f}\left[h^{1 / 2} K_{h}\left(\theta-\Theta_{1}\right)\right]<\infty$ from $(22)$.

Since (20) satisfies the condition of Lindeberg (Feller (1968, p.244)) from (21) and $(22)$, it is given as the follows,

$$
\sqrt{n h}\left\{\hat{f}_{h}(\theta)-\mathrm{E}_{f}\left[\hat{f}_{h}(\theta)\right]\right\} \stackrel{d}{\longrightarrow} \mathrm{N}(0, f(\theta) / \pi), \quad n \rightarrow \infty .
$$

The order of the second term of (19) is equal to,

$$
\begin{aligned}
\sqrt{n h} \operatorname{bias}\left[\hat{f}_{h}\right] & =\sqrt{n h} O(h) \\
& =O\left(\sqrt{n h^{3}}\right) .
\end{aligned}
$$

with $h=c n^{-\alpha}$, we obtain the follows,

$$
\begin{aligned}
\sqrt{n h^{3}} & \sim n^{1 / 2} n^{-3 \alpha / 2} \\
& =n^{(1-3 \alpha) / 2} .
\end{aligned}
$$

When $\alpha>1 / 3$ is chosen, (24) is given as the following form:

$$
\begin{aligned}
\sqrt{n h} \operatorname{bias}\left[\hat{f}_{h}(\theta)\right] & =O\left(\sqrt{n h^{3}}\right) \\
& =o(1)
\end{aligned}
$$

For $\alpha>1 / 3$ and as $n \rightarrow \infty$, Theorem 3 completes the proof from (23) and (25). 


\section{References}

Davis, K. B. (1975). Mean Square Error Properties of Density Estimates, The Annals of Statistics 3, 1025-1030.

Di Marzio, M., Panzera, A. and Taylor, C. C. (2009). Local polynomial regression for circular predictors, Statistics \& Probability Letters, 79, 2066-2075.

Di Marzio, M., Panzera, A. and Taylor, C. C. (2011). Kernel density estimation on the torus, Journal of Statistical Planning and Inference, 141, 2156-2173.

Feller, W. (1968). An Introduction to Probability Theory and Its Applications. I (Third ed.). John Wiley \& Sons, Inc, 244.

Garcia-Portugues, E. (2013). Exact risk improvement of bandwidth selectors for kernel density estimation with directional data, Electronic Journal of Statistics, 7, 16551685 .

Hall, P., Watson, G. S. and Cabrera, J. (1987). Kernel density estimation with spherical data, Biometrika, 74, 751-762.

Mardia, K. V. and Jupp, P. E. (1999). Directional Statistics, Wiley.

Taylor, C. C. (2008). Automatic bandwidth selection for circular density estimation, Computational Statistics \& Data Analysis, 52, 3493-3500 .

Scott, D. W. (1992). Multivariate Density Estimation: Theory, Practice, and Visualization, Wiley.

Received June 11, 2016

Revised January 16, 2017 